\title{
Low-Profile Circularly Polarized Array with Gain Enhancement and RCS Reduction Using Polarization Conversion EBG Structures
}

\author{
Qi Zheng, Chenjiang Guo, Guy A. E. Vandenbosch, and Jun Ding
}

\begin{abstract}
A planar antenna array based on checkerboard polarization conversion electromagnetic band-gap (EBG) structures with wideband circular polarization and low radar cross section (RCS) is presented. The array is formed by $2 \times 2$ circularly polarized (CP) element antennas fed with a sequential feeding network. On the one hand, CP radiation is obtained due to the polarization conversion characteristic of the metamaterials. A sequentially rotated feeding network is employed for wide operating bandwidth, polarization purity and radiation pattern symmetry. Measured results show that the array has a wide usable bandwidth of $18.4 \%$, the common part of an impedance bandwidth of $37.3 \%(4.80-7.00 \mathrm{GHz}$ ), a 3-dB axial ratio (AR) bandwidth of $22.5 \%$ (5.00-6.27 GHz) and a 3-dB gain bandwidth of 18.4\% (4.94-5.94 GHz). On the other hand, the checkerboard arrangement of the EBG structure generates both an in-band and a partial out-band RCS reduction under normal incidence for both $x$ - and $y$-polarizations. Besides, the proposed array achieves a significant gain enhancement compared to the traditional slot array. The experimental and simulated results are in good agreement.
\end{abstract}

Index Terms-Checkerboard polarization conversion EBGs (CPC EBGs), radar cross section (RCS), circular polarization, wideband, antenna array

\section{INTRODUCTION}

Polarization manipulation based on metamaterials has attracted a growing interest. This phenomenon may play an important role in microwave, $\mathrm{THz}$ and optical engineering areas [1-4]. One important application is circularly polarized (CP) antennas. For example, a Fabry-Perot (F-P) cavity antenna with a partially reflective surface was proposed to achieve circular polarization with a linearly polarized (LP) feed [5]. A novel polarization rotation technique based on an artificial magnetic conductor (AMC) was utilized in a low-profile dipole antenna for the generation of circular polarization [6]. Recently, metamaterials with polarization conversion properties have also been designed in combination with traditional slot antennas [7-9].

Another important application is to reduce radar cross sections (RCS) [10], [11]. A checkerboard arrangement of polarization conversion metasurfaces (PCMs) has the capability to reduce RCSs of

Manuscript received June 29, 2019. This work was supported by the China Scholarship Council (No. 201806290108).

Q. Zheng is with the School of Electronics and Information, Northwestern Polytechnical University, Xi'an China, and also with the ESAT-TELEMIC Research Division, Department of Electrical Engineering, KU Leuven, 3001 Leuven, Belgium. (Corresponding author, e-mail: zhq930908@mail.nwpu.edu.cn).

C. Guo, and J. Ding are with the School of Electronics and Information, Northwestern Polytechnical University, Xi'an China. (e-mail: cjguo@nwpu.edu.cn, dingjun@nwpu.edu.cn).

G. A. E. Vandenbosch is with the ESAT-TELEMIC Research Division, Department of Electrical Engineering, KU Leuven, 3001 Leuven, Belgium. (e-mail: guy.vandenbosch@esat.kuleuven.be).

Color versions of one or more of the figures in this communication are available online at http://ieeexplore.ieee.org.

Digital Object Identifier 10.1109/TAP.2016.xxx objects. They can for example be used in low RCS antennas [12-18]. More specifically, in [15] checkerboard PCMs and circular polarization techniques are combined to design a $\mathrm{CP}$ antenna with low RCS over a wide band. The antenna achieves a significant gain enhancement by using a F-P cavity. However, the antenna requires a high volume due to the distance between the source and the superstrate. Many attempts have been made to design low-profile and low RCS antennas [19-23]. For instance, a low RCS CP antenna array based on an orthogonal arrangement of metamaterials-based polarization dependent elements was proposed in [19]. The array shows a $17 \%$ operating bandwidth, covering both $\mathrm{S} 11<-10 \mathrm{~dB}$ and $\mathrm{AR}<3 \mathrm{~dB}$, with a remarkable wideband $\mathrm{RCS}$ reduction.

In this paper, a planar $\mathrm{CP}$ antenna array based on simple corner-truncated EBGs with a total thickness of $0.05 \lambda_{0}\left(\lambda_{0}\right.$ is the operating wavelength in free space) is proposed. Owing to the polarization conversion characteristic of the EBGs and the bandwidth widening property of the sequential rotation technique [24], [25], the array obtains a wideband $\mathrm{CP}$ radiation as well as a wideband RCS reduction and significant gain enhancement compared to the traditional slot array. The performance is studied numerically and verified experimentally. The paper is organized as follows. Section II presents the configuration and the theoretical analysis of the proposed array. Section III gives the measured radiation and scattering properties. Section IV concludes the paper.

\section{DESIGN AND ANALYSIS OF THE ARRAY}

\section{A. Configuration of the array}

The configuration of the proposed array is displayed in Fig. 1. It is comprised of $2 \times 2$ elements fed by a sequentially rotation feeding network. The element is a traditional slot antenna loaded with $4 \times 4$ polarization conversion EBG unit cells. The feeding network with a phase distribution of $0^{\circ}, 90^{\circ}, 180^{\circ}$ and $270^{\circ}$ is designed with three $\mathrm{T}$-junction sections, including one anti-phase delay line and two $90^{\circ}$ phase delay lines.

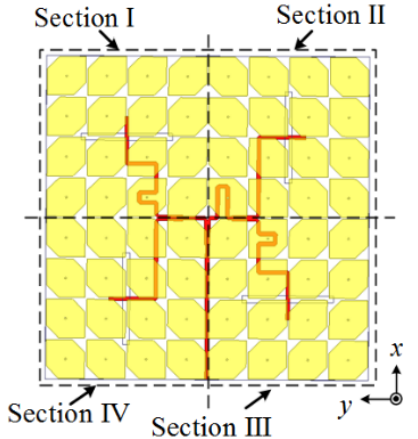

(a)

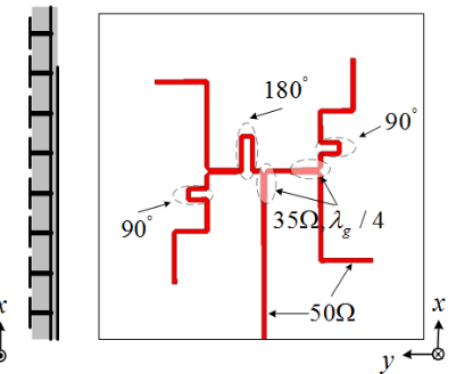

(b) (c)
Fig.1 Configuration of the array, (a) top view, (b) side view and (c) back view

\section{B. Design and analysis of array element}

As shown in Fig. 2, the array element is comprised of $4 \times 4$ EBG 
unit cells, a slotted ground plate and a microstrip feeding line separated by two substrates with a relative dielectric constant of 3.5 and a loss tangent of 0.003 . The unit cell is a simple corner-truncated EBG structure, which consists of a corner-truncated square patch and a grounded shorting via. The via is located at the centre of the cell. The unit cells are distributed periodically in the centre of the substrate.

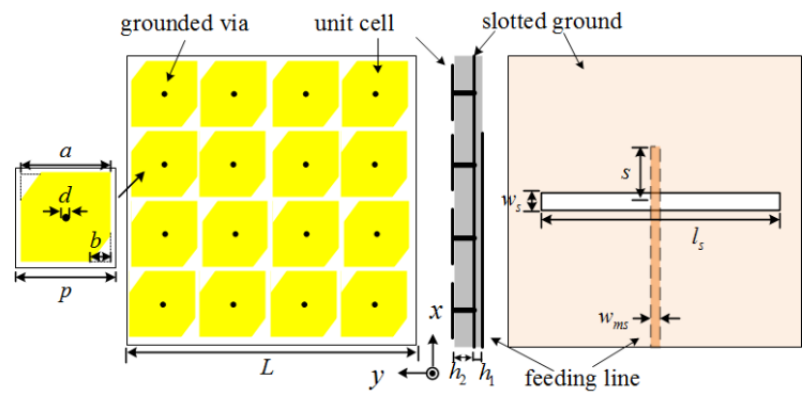

Fig. 2 Geometry of element in the array.

As shown in Fig. 3, the $\mathrm{CP}$ radiation performance of the element can be schematically explained using an equivalent circuit model [7]. A linearly polarized wave radiated from the slot along the $x$-axis can be decomposed into two orthogonal components along the diagonal directions. Due to the presence of the truncated corners in the patches, $\mathrm{CP}$ radiation can be ensured by adjusting the truncated corner size. The unit cell was optimized targeting a wide $3-\mathrm{dB}$ AR bandwidth using HFSS, yielding: $p=11.5 \mathrm{~mm}, a=11 \mathrm{~mm}, b=3.5 \mathrm{~mm}, d=0.6 \mathrm{~mm}$, $L=46 \mathrm{~mm}, l_{s}=26 \mathrm{~mm}, w_{s}=2 \mathrm{~mm}, l_{f}=7 \mathrm{~mm}, w_{m s}=1.15 \mathrm{~mm}$, $h_{1}=0.5 \mathrm{~mm}, h_{2}=2 \mathrm{~mm}$.

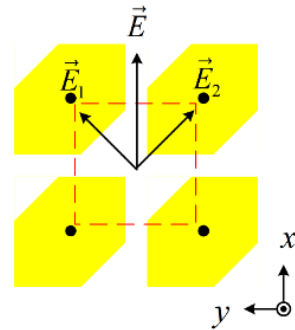

(a)

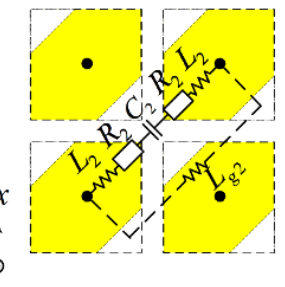

(b)

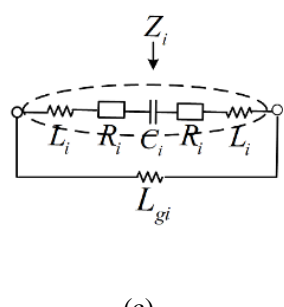

(c)
Fig. 3 (a) New unit cell, (b) analysis diagram of the equivalent circuit model, (c) the final equivalent circuit. $C_{i} \quad(i=1,2)$ are generated by the gaps between two adjacent patches along the diagonal directions. $L_{g i}$ is the inductance due to the two shorting vias and the current path on the ground plane in between.

The schematics of a traditional LP slot antenna (Ant. 1), a LP slot antenna with a square patch (Ant. 2), and a CP slot antenna with via-less EBGs (Ant. 3) are displayed in Fig. 4. The performances of the three antennas and the proposed antenna (Ant. 4) are compared in Fig. 5 to demonstrate the advantages of the proposed structure. Note that corresponding structural parameters (concerning substrates, microstrip line, slot, etc.) as much as possible are kept the same in the four antennas. The square patch with dimensions $31.5 \mathrm{~mm} \times 31.5 \mathrm{~mm}$ in Ant. 2 is selected to ensure the same operating frequency of $5.5 \mathrm{GHz}$.

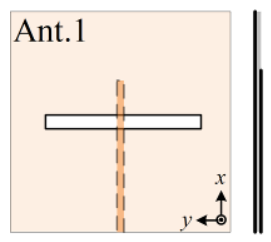

(a)

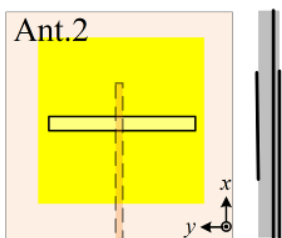

(b)

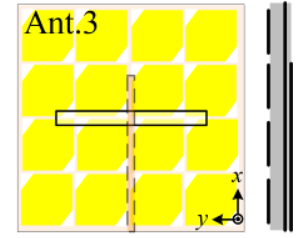

(c)
Fig. 4 Schematics of (a) Ant. 1, (b) Ant. 2, and (c) Ant. 3.

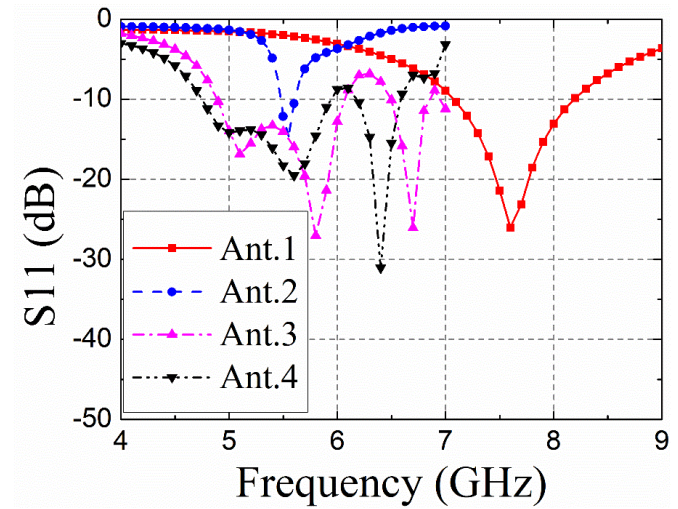

(a)

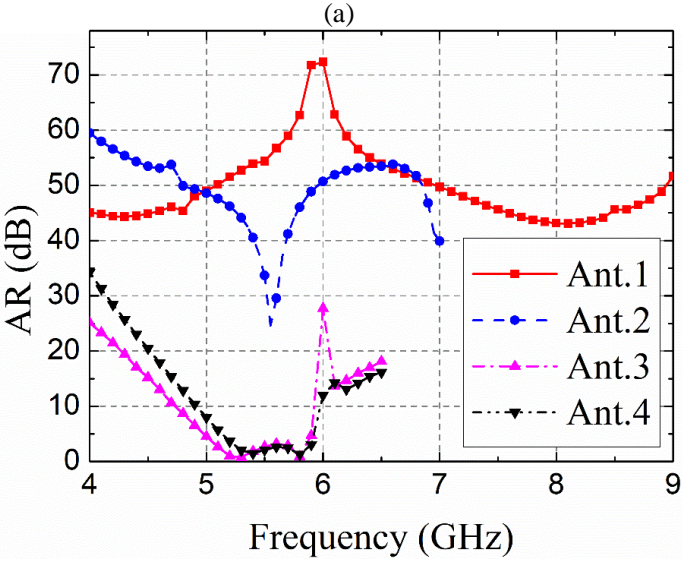

(b)

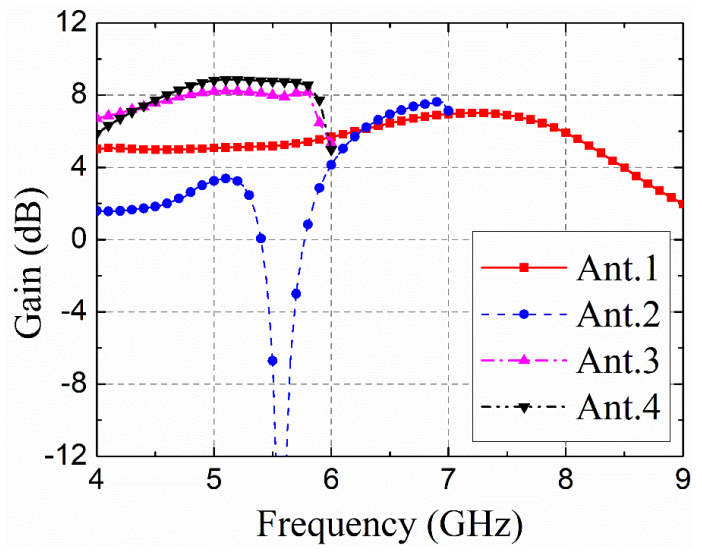

(c)

Fig. 5 Comparison of (a) S11, (b) AR, and (c) gain versus frequency

Figs. 5 (a) and (b) give the impedance bandwidth and AR characteristic of the four antennas. It is shown that Ant. 1 is a LP antenna operating from $7.05 \mathrm{GHz}$ to $8.05 \mathrm{GHz}$. One simple solution to obtain an antenna operating at the center frequency of $5.5 \mathrm{GHz}$ is to integrate the Ant. 1 with a square patch, as depicted in Ant. 2. However, Ant. 2 operates in a narrow band. Another method is integrating corner-truncated via-less EBGs (Ant. 3) or corner-truncated EBGs with vias (Ant. 4), which shifts the impedance bandwidths significantly to a lower frequency region. This is due to the additional capacitance and inductance caused by the superstrate. CP radiation can be obtained for both Ant. 3 and Ant. 4 [9]. Further, according to Fig. 5(c), both Ant. 3 and Ant. 4 achieve a gain that is stable within the operating bandwidth, and that is significantly higher (over $3 \mathrm{~dB}$ ) than for Ant. 1 and Ant. 2. Comparing Ant. 3 and Ant. 4, it can be viewed that Ant. 4 achieves an average $0.5 \mathrm{~dB}$ higher gain compared to the via-less EBG-based Ant. 3 [26]. 
Fig. 6 gives the radiation patterns of the element antenna at $5.5 \mathrm{GHz}$. The antenna has a LHCP radiation and nearly symmetric radiation patterns in both xoz-plane and yoz-plane. The cross-polarization levels are lower that $-20 \mathrm{~dB}$ in the two planes, which can be a good candidate for array design.

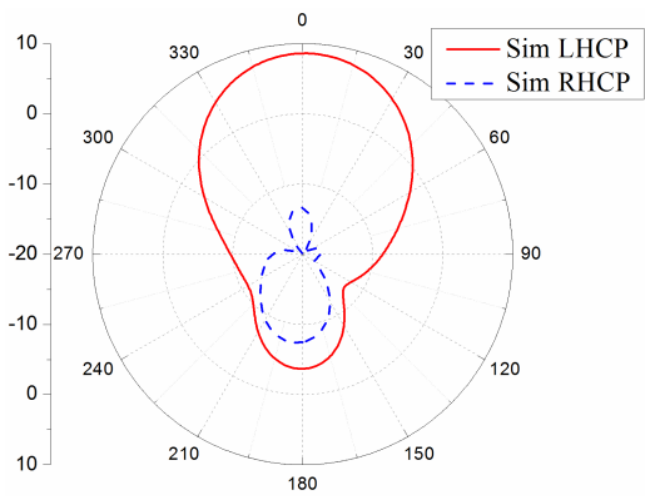

(a)

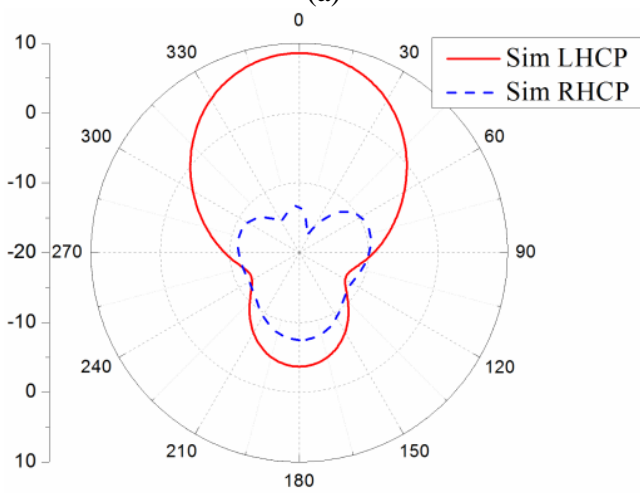

(b)

Fig. 6 Radiation pattern of the element antenna at $5.5 \mathrm{GHz}$, (a) xoz-plane, (b) yoz-plane

The schematics of an array with $2 \times 2$ traditional LP slot element antennas (Array with Ant. 1) and an array with $2 \times 2$ LP slot patch antennas ( Array with Ant. 2) are displayed in Fig. 7. Fig. 8 compares the proposed array and the arrays with Ant. 1 and Ant. 2 as elements via simulations. The array with Ant. 1 as element has an impedance bandwidth from 4.75 to $5.90 \mathrm{GHz}$ and a gain from 5.03 to $6.75 \mathrm{~dB}$. The array with Ant. 2 as element operates in the band 5.45-5.95 GHz with an average gain of $1.2 \mathrm{~dB}$. The proposed array has an impedance bandwidth from 4.70 to $6.95 \mathrm{GHz}$ and a 3-dB AR from 4.90 to 6.05 $\mathrm{GHz}$ with an average gain of $10.5 \mathrm{~dB}$ in simulation. The maximum gain of $12.72 \mathrm{~dB}$ occurs at $5.60 \mathrm{GHz}$. It can be concluded that the proposed array has a significantly higher gain compared to the array with traditional slot Ant. 1 as elements and patch Ant. 2 as elements.

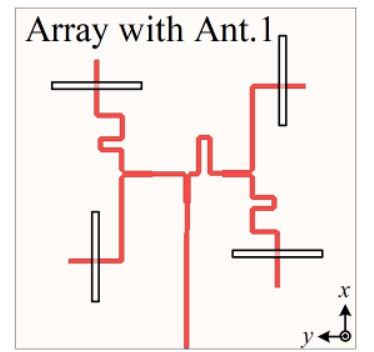

(a)

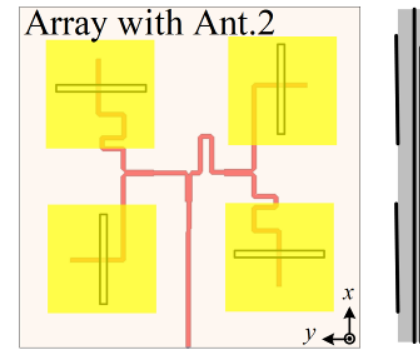

(b)
Fig. 7 Schematics of (a) Array with Ant. 1, and (b) Array with Ant. 2.

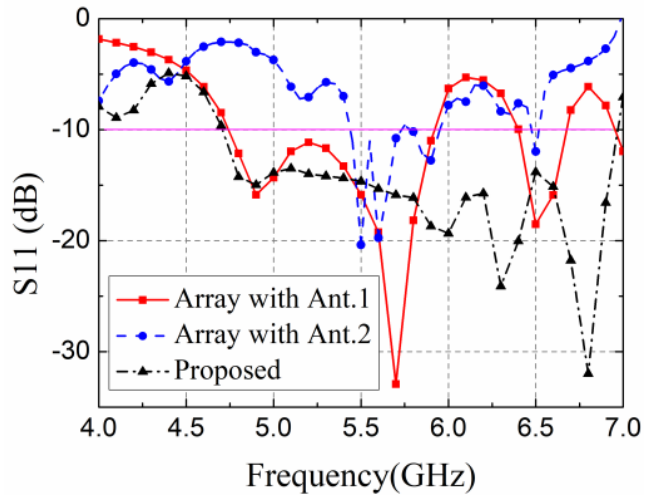

(a)

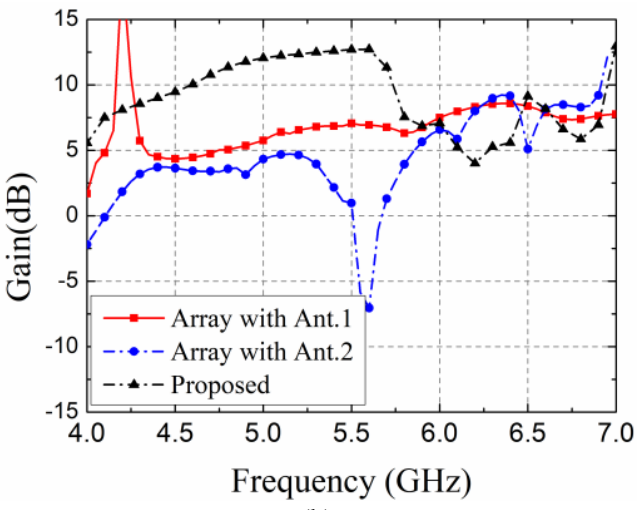

(b)

Fig. 8 Simulated results of (a) S11 and (b) radiation gain.

\section{RCS reduction}

The array can also be viewed as consisting of four rotated sections (Section I, II, III and IV, as shown in Fig. 1(a)) forming a checkerboard consisting of $8 \times 8$ polarization conversion EBG unit cells. To analyse RCS reduction, first the polarization conversion property of the EBG unit cell is analysed. The simulation model in HFSS is presented in Fig. 9. Fig. 10(a) shows the reflection coefficient for a normally impinging plane wave along the $-z$ direction. The cross-polarization reflection coefficient is above $-3 \mathrm{~dB}$ from 4.70 to $5.90 \mathrm{GHz}$, which means that a LP incident wave is mainly converted into a reflected wave with orthogonal polarization. It can be seen from Fig. 10(b) that the phase difference between the EBG unit cells in Section I and the ones in Section II is $180^{\circ}$. Therefore, as shown in Fig. $10(\mathrm{c})$, taking an $x$-polarized incident wave $\left(\vec{E}_{x}^{i}\right)$ as an example, the $y$-component (cross-polarization $\vec{E}_{c r o}^{r}$ ) of the reflected fields of the four rotated sections will be cancelled out and only the $x$-component (co-polarization $\vec{E}_{c o}^{r}$ ) is left. According to the definition of the Radio Cross Section (RCS) of a target $\sigma=4 \pi \lim _{R \rightarrow \infty} R^{2}\left(\left|E_{s}\right|^{2} /\left|E_{i}\right|^{2}\right)$ [27] (where $\mathrm{R}$ is the distance between the target and the observation point, and $E_{i}$ and $E_{s}$ are the electric field of the incident and scattered waves), the total field reflected by the four rotated sections can be significantly reduced. 


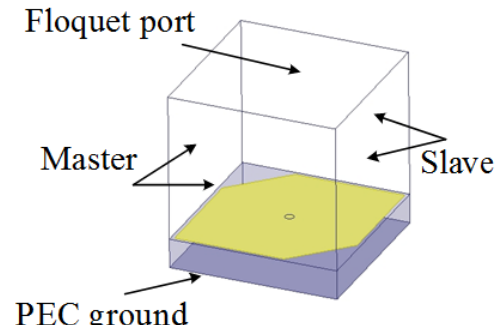

(a)

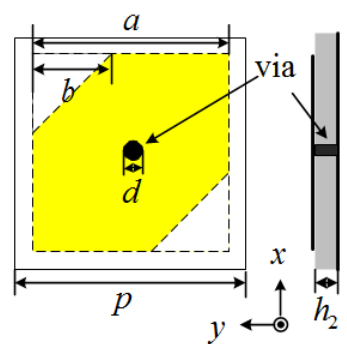

(b)
Fig. 9 Topology of the unit cell, (a) simulation model, (b) top view and side

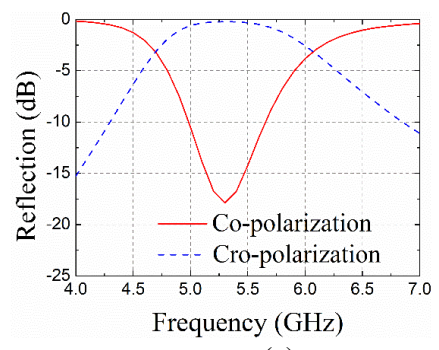

(a)

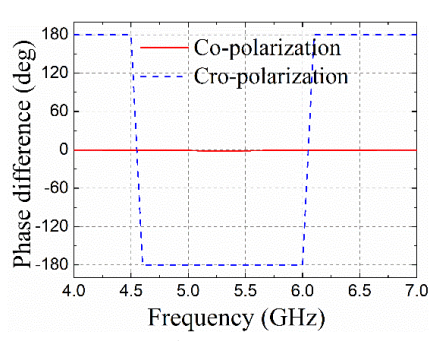

(b)

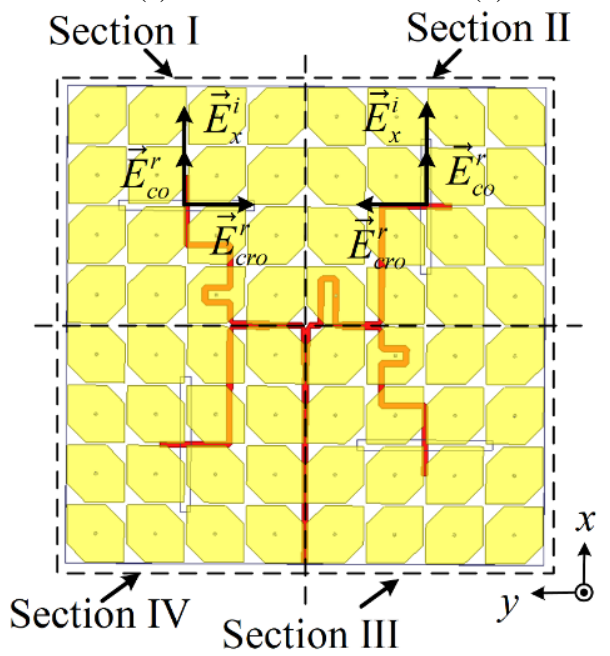

(c)

Fig.10 (a) Simulated reflection coefficient, (b) phase difference between adjacent sections and (c) reflected field under $x$-polarized incident wave

\section{MEASUREMENT AND DISCUSSIONS}

In order to verify the topology, a prototype was fabricated and measured, see Fig. 11. The overall size is $92.0 \mathrm{~mm} \times 92.0 \mathrm{~mm} \times 2.5$ $\mathrm{mm}\left(1.81 \lambda_{0} \times 1.81 \lambda_{0} \times 0.05 \lambda_{0}\right)$. The antenna is fed with a $50 \Omega$ SMA connector. Measurements were performed with an Agilent PNA Series Network Analyzer E8363B.

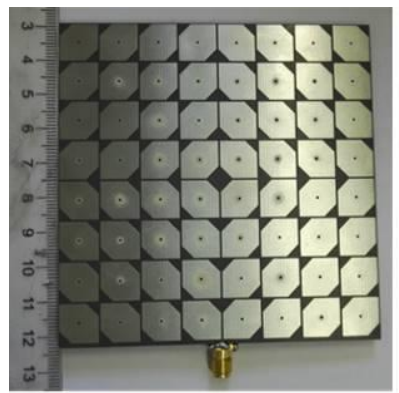

(a)

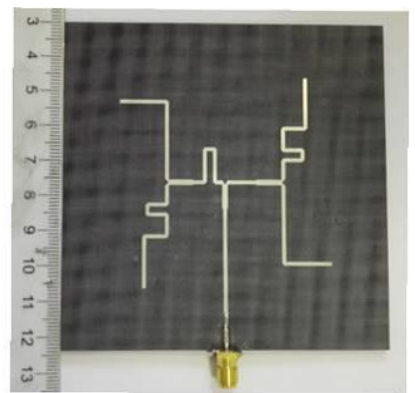

(b)
Fig. 11 Fabricated prototype, (a) top view, (b) bottom view
Radiation performance and scattering properties were measured in an anechoic room. The measured results agree well with the simulated ones. As seen from Fig. 12, the array has a measured impedance bandwidth of $37.3 \%$, from 4.80 to $7.00 \mathrm{GHz}$, a 3-dB AR bandwidth of $22.5 \%$, from 5.00 to $6.27 \mathrm{GHz}$, and a 3 -dB gain bandwidth of $18.4 \%$, from 4.94 to $5.94 \mathrm{GHz}$. The maximum gain of $13.00 \mathrm{dBic}$ is obtained at $5.60 \mathrm{GHz}$. As seen from Fig. 13, the radiation pattern at $5.50 \mathrm{GHz}$ is symmetric and the cross-polarization levels are lower than $-20 \mathrm{~dB}$ in both the xoz- and yoz-planes. The acceptable differences between the measured and simulated results are attributed to fabrication and measurement errors. The simulated radiation efficiency is over $80 \%$ in the entire bandwidth.

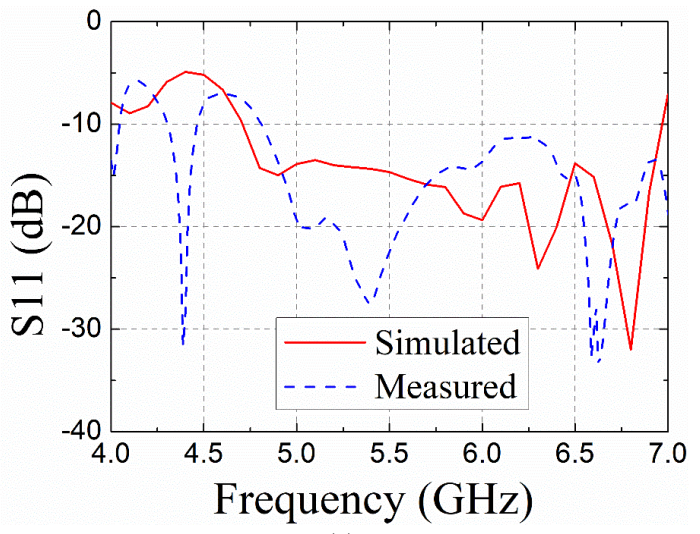

(a)

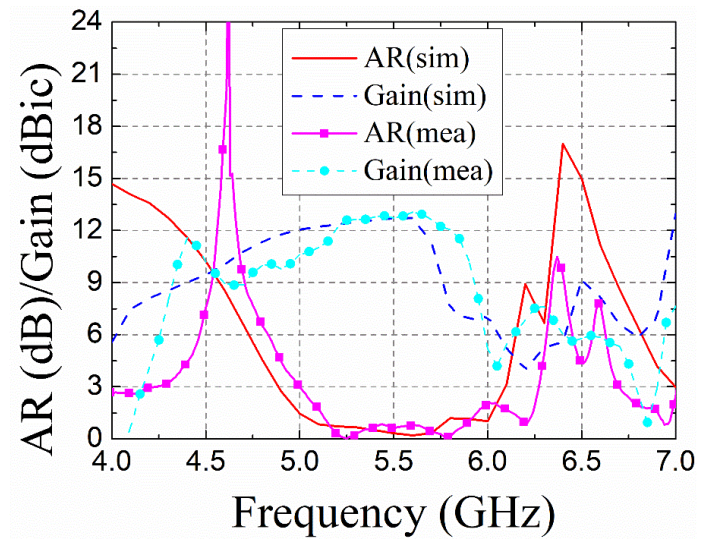

(b)

Fig. 12 (a) Reflection coefficient (b) AR and gain

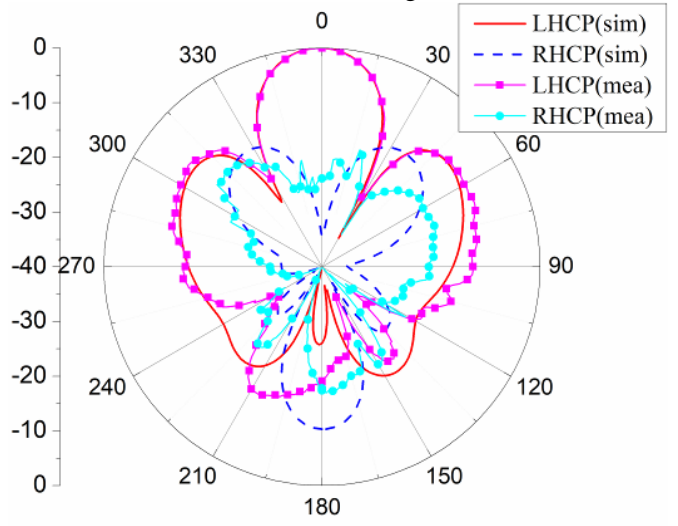

(a) 


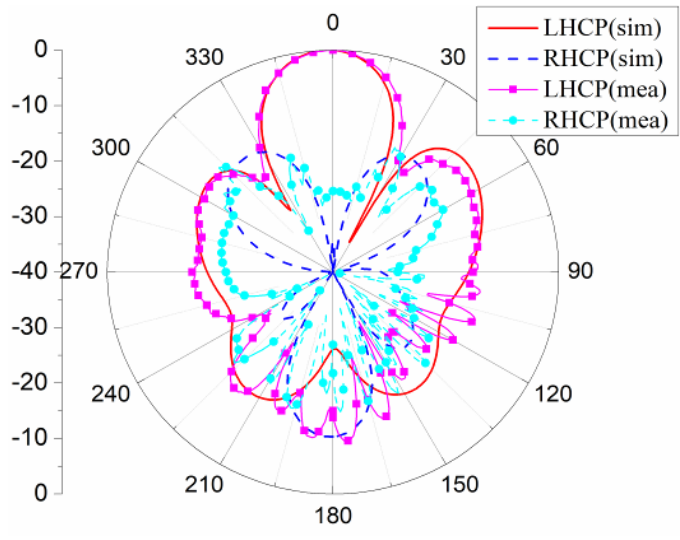

(b)

Fig. 13 Normalized radiation patterns of the array at $5.50 \mathrm{GHz}$, (a) xoz-plane, (b) yoz-plane

To demonstrate the effectiveness of a checkerboard polarization conversion electromagnetic band-gap structure (CPC EBGs) on RCS reduction, the simulated and measured RCS's of the proposed array and the array of $2 \times 2$ slot antennas without CPC EBGs (Reference array) are compared. Fig. 14(a) gives a schematic view of the measurement setup. Two LP horn antennas serve as transmitter and receiver in the measurement setup in an anechoic chamber. The incident wave generated by the transmit antenna can be viewed as a plane wave. The reflected field can be observed by the receive antenna. As shown in Fig. 14(b), the proposed array obtains an over $6 \mathrm{~dB}$ RCS reduction from 4.70 to $5.80 \mathrm{GHz}$ for both the $x$ - and $y$-polarization. The maximum RCS reduction occurs at $5.10 \mathrm{GHz}$ for both polarizations. The results are in accordance with the polarization conversion band and resonant frequency given in Fig. 10(a). The small frequency shift may be due to edge diffraction. The discrepancies between measured and simulated results are acceptable considering fabrication tolerances and measurement deviations due to for example antenna misalignment. Fig. 15 presents the monostatic RCS for different incident angels at $5.10 \mathrm{GHz}$ for the $x$-polarized wave (incident field $\vec{E}$ parallel to the $x$-axis in xoz-plane) and the $y$-polarized wave (incident field $\vec{E}$ parallel to the $y$-axis in yoz-plane), which shows the remarkable RCS reduction realized within an angular range of about 40 degrees.

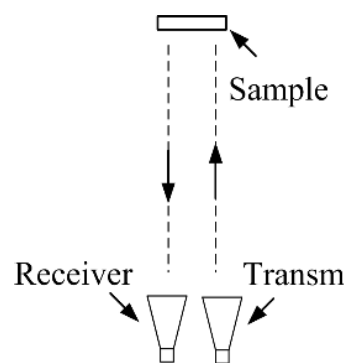

(a)

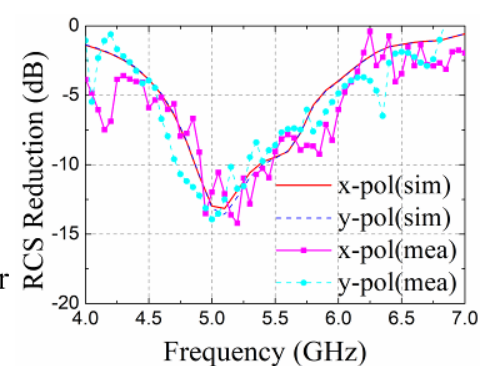

(b)
Fig.14 (a) Schematic view of measurement setup (b) Measured and simulated RCS reduction of the array with respect to the array without CPC EBGs under normal incidence

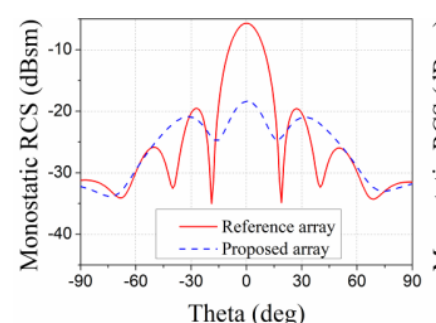

(a)

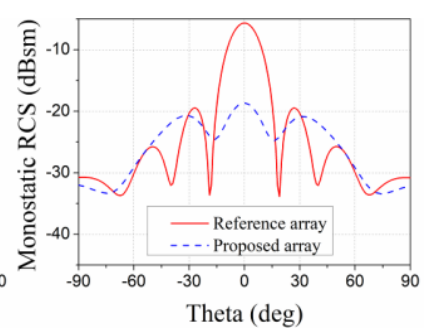

(b)
Fig. 15 Simulated monostatic RCS at $5.10 \mathrm{GHz}$ as a function of incidence angle for (a) $x$-polarized and (b) $y$-polarized impinging waves

A comparison between the proposed design and low-RCS metasurface-based antennas in literature is given in Table I. The proposed array has the largest operating bandwidth, the smallest size, and a comparable gain enhancement and $3-\mathrm{dB}$ gain bandwidth.

TABLE I. COMPARISON WITH LOW-RCS METAMATERIAL-BASED ANTENNAS IN LITERATURE

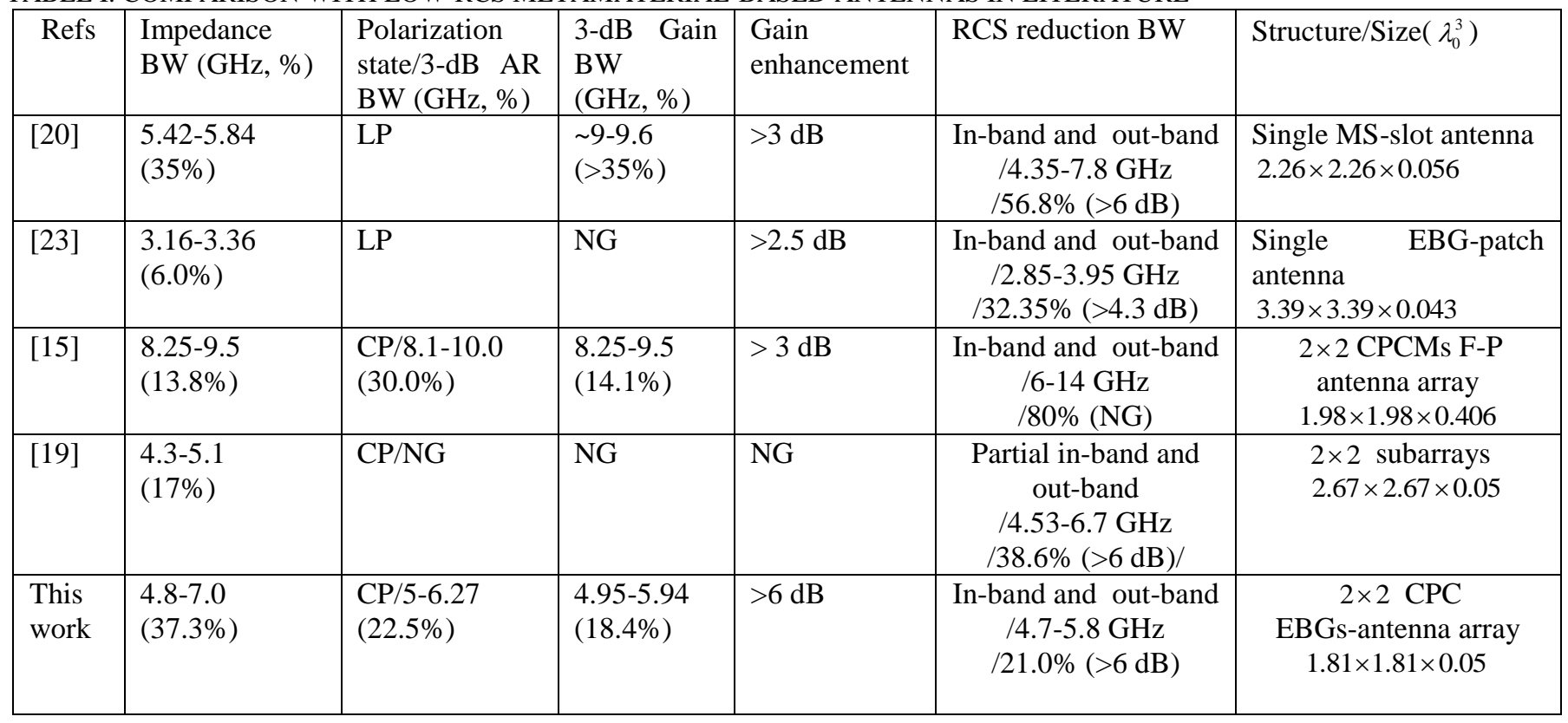




\section{CONCLUSION}

In this paper, a low-profile $\mathrm{CP}$ antenna array with wideband gain enhancement and RCS reduction was realized by placing polarization conversion EBG units in an $8 \times 8$ checkerboard topology. Owing to the polarization conversion characteristic of the corner-truncated EBG unit cell, the low-profile array has a 3-dB AR bandwidth of $22.5 \%$ with both an in-band and a partial out-band RCS reduction. Besides, the proposed array achieves a wide $3-\mathrm{dB}$ gain bandwidth of $18.4 \%$ with a maximum of $13 \mathrm{~dB}$. Good agreement between simulated and measured results was observed. The proposed array can find application in satellite communications and stealth aircrafts.

\section{REFERENCES}

[1] H. Chen, et al., "Ultra-wideband polarization conversion metasurfaces based on multiple plasmon resonances," J Appl Phys, vol. 145, no. 15, p. 154504, 2014.

[2] Q. Zheng, et al., "Wideband metasurface-based reflective polarization converter for linear-to-linear and linear-to-circular polarization conversion," IEEE Antennas Wireless Propag. Lett., vol.17, no. 8, pp. 1459-1463, 2018.

[3] N.K. Grady, et al., "Terahertz metamaterials for linear polarization conversion and anomalous refraction," Science, vol. 340, no. 6138, pp. 1304-1307, 2013.

[4] J. Hao, et al., "Optical metamaterial for polarization control," Phys Rev A, vol. 80, no. 2, p. 023807, 2009.

[5] R. Orr, G. Goussetis, and V. Fusco, "Design method for circularly polarized Fabry-Perot cavity antennas," IEEE Trans Antennas Propag, vol. 62 , no. 1, pp. 19-26, 2014.

[6] W. Yang, et al., "Novel polarization rotation technique based on an artificial magnetic conductor and its application in a low-profile circular polarization antenna," IEEE Trans. Antennas Propag., vol. 62, no. 12, pp. 6206-6216, 2014.

[7] H.L. Zhu, et al., "Design of polarization reconfigurable antenna using metasurface," IEEE Trans Antennas Propag., vol. 62, no. 6, pp. 2891-2898, 2014.

[8] Ta, Son Xuat, and Ikmo Park. "Planar wideband circularly polarized metasurface-based antenna array," Journal of Electromagnetic Waves and Applications, vol. 12, no. 12, pp. 1620-1630, 2016.

[9] Y. Huang, et al., "Polarization conversion of metasurface for the application of wide band low-profile circular polarization slot antenna," Appl. Phys. Lett., vol. 109, no. 5, p. 054101, 2015.

[10] Y. Jia, et al., "A dual-patch polarization rotation reflective surface and its application to ultra-wideband RCS reduction," IEEE Trans. Antennas Propag., vol. 65, no. 6, pp. 3291-3295, 2017.

[11] Q. Zheng, et al., "Broadband radar cross section reduction using polarization conversion metasurface," International Journal of Microwave and Wireless Technologies, vol. 10, no. 2, pp. 197-206, 2018.
[12] Y. Liu, et al., "Wideband RCS reduction of a slot array antenna using polarization conversion metasurfaces," IEEE Trans. Antennas Propag., vol. 64, no. 1, pp. 326-331, 2015.

[13] L. Zhang and T. Dong, "Low RCS and high-gain CP microstrip antenna using SA-MS," Electron Lett., vol. 53, no. 6, pp. 375-376, 2017.

[14] M. Long, W. Jiang, and S.X. Gong, "Wideband RCS reduction using polarization conversion metasurface and partially reflecting surface," IEEE Antennas Wireless Propag. Lett., vol. 16, pp. 2534-2537, 2017.

[15] K. Li, et al., "A circularly polarized high-gain antenna with low RCS over a wideband using chessboard polarization conversion metasurfaces," IEEE Trans. Antennas Propag., vol. 65, no. 8, pp. 4288-4292, 2017.

[16] Q. Zheng, C. Guo, and J. Ding, "Wideband and low RCS planar circularly polarized array based on polarization conversion of metasurface," Microw Opt Techn Let, vol. 60, no. 3, pp. 784-789, 2018.

[17] Q. Chen, and H. Zhang. "High-gain circularly polarized Fabry-Pérot patch array antenna with wideband low-Radar-Cross-Section property," IEEE Access, 7, pp. 8885-8889, 2019.

[18] S. Zarbakhsh, et al., "Broadband and high-gain circularly-polarized antenna with Low RCS," IEEE Trans. Antennas Propag., vol. 67, no. 1, pp. 16-23, 2019.

[19] Y. Zhao, et al., "Broadband low-RCS circularly polarized array using metasurface-based element," IEEE Antennas Wireless Propag. Lett., vol. 16, pp. 1836-1839, 2017.

[20] Y. Zhao, et al., "Broadband low-RCS metasurface and its application on antenna," IEEE Trans. Antennas Propag., vol. 64, no. 7, pp. 2954-2962, 2016.

[21] Y. Liu, et al., "Radar cross section reduction of a microstrip antenna based on polarization conversion metamaterial," IEEE Antennas Wireless Propag. Lett., vol. 15, pp. 80-83, 2015.

[22] Y. Zhou, et al., "In-band RCS reduction and gain enhancement of a dual-band PRMS-antenna," IEEE Antennas Wireless Propag. Lett., vol. 1, pp. 2716-2720, 2017.

[23] Z. J. Han, W. Song, and X. Q. Sheng, "Gain enhancement and RCS reduction for patch antenna by using polarization-dependent EBG surface," IEEE Antennas Wireless Propag. Lett., vol. 16, pp. 1631-1634, 2017.

[24] H. Evans, et al., "Application of simulated annealing to design of serial feed sequentially rotated $2 \times 2$ antenna array," Electron. Lett, vol. 36, no. 24, pp. 1987-1988, 2000.

[25] K.K. Pang, et al., "Circularly polarized dielectric resonator antenna subarrays," Microw Opt Techn Let, vol. 27, no. 6, pp. 377-379, 2000.

[26] W. Liu, Z.N. Chen, and X. Qing, Metamaterial-based low-profile broadband mushroom antenna, IEEE Trans. Antennas Propag., vol. 62, no. 3, pp. 1165-1172, 20113.

[27] E. F. Knott, J. F. Shaeffer, and M. T. Tuley, Radar Cross Section. Raleigh, NC, USA: SciTech, 2004. 